\title{
Therapie des Bronchialkarzinoms: Interdisziplinär und mit Expertise
}

\section{Thomas}

\author{
Therapy of Bronchial Carcinoma: Interdisciplinary and with Expertise
}

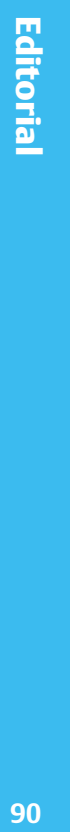

Das Bronchialkarzinom ist die häufigste krebsbedingte Todesursache überhaupt. Allein in Deutschland werden jedes Jahr 40000 Erkrankungsfälle neu diagnostiziert. Jährlich sterben mehr Menschen am Bronchialkarzinom als an Brustkrebs, Dickdarmkrebs und dem Prostatakarzinom zusammengenommen [1]. Die 5-Jahres-Überlebensrate ist mit $13 \%$ seit Jahren nicht günstig und hat das ärztliche Engagement bei der Therapie dieser Erkrankung immer wieder enttäuscht.

In der Sektion „Pneumologische Onkologie“ der Deutschen Gesellschaft für Pneumologie (DGP) wurde in den letzten Jahren häufig zu den Fragen der optimalen stadienbezogenen Therapieformen des Bronchialkarzinoms und der Tatsache, dass diese von der Breite der Behandler nicht immer für alle Patienten vorgeschlagen werden, diskutiert. In diesen Diskussionen wurde deutlich, dass bislang die Chance einer optimalen Therapie für Patienten mit Bronchialkarzinomen in den entsprechenden Zentren am besten möglich ist. Im Kontext dieser Diskussion ist der Gedanke zur Konzeption einer „Empfehlung“ zur Therapie des Bronchialkarzinoms entstanden. Eine Empfehlung, die an den behandelnden Arzt gerichtet ist und durch eine detaillierte und evidenzgestützte Beschreibung der Therapiealgorithmen auch zur Vorstellung und Behandlung geeigneter Patienten in entsprechenden Zentren führen soll [2].

Die Berücksichtigung von Begleiterkrankungen und der individuellen Situation des Patienten ist bei der Behandlung eines Bronchialkarzinoms grundlegend. Die Behandlung selbst ist interdisziplinär ausgerichtet und gerade in multimodalen Therapiekonzepten ist die Diskussion zwischen den beteiligten Fachdisziplinen entscheidend [3]. Mit Vertretern aller an der Behandlung des Bronchialkarzinoms beteiligten Fachgesellschaften hat auf Initiative der Sektion „Pneumologische Onkologie“ eine Autoren- gruppe mit der Erstellung der in diesem Heft der PNEUMOLOGIE gedruckten Empfehlung begonnen. Aufgabe der beteiligten Autoren war es, jeweils einen Manuskriptteil evidenzbasiert zu erstellen. Jeder Manuskriptteil wurde dann in der Autorengruppe kritisch kommentiert. Diese Kommentare waren dann jeweils Anregung für den betreffenden Autor zur weiteren Überarbeitung. Im nächsten Schritt erfolgte dann die Bearbeitung der Teilmanuskripte zu einem Gesamtmanuskript durch den federführenden Autor. Das so entstandene Manuskript wurde erneut in der Autorengruppe zirkuliert und kommentiert. Zur Bearbeitung der Kommentare fanden Treffen der Autorengruppe statt, in denen diese Kommentare, Punkt-für-Punkt, strukturiert diskutiert wurden. Das Ergebnis führte dann zu einer vorläufig abschließenden Manuskriptrevision. Dieses Manuskript wurde von allen beteiligten Fachgesellschaften begutachtet und erforderliche Revisionen im Konsens der Autorengruppe eingearbeitet.

In diesem Heft der PNEUMOLOGIE (siehe S. 113) kommt nunmehr die gemeinsam von der DGP, der Deutschen Gesellschaft für Thoraxchirurgie (DGT), der Deutschen Gesellschaft für Radioonkologie (DEGRO) und der Arbeitsgemeinschaft für Internistische Onkologie in der Deutschen Krebsgesellschaft (AIO) getragene Empfehlung zur Therapie des Bronchialkarzinoms zur Publikation.

\section{Literatur}

\footnotetext{
${ }^{1}$ Landis SH, Murray T, Bolden S, Wingo P. Cancer statistics. Cancer J Clin 1998; 48: 6-29

2 Bach P, Cramer L, Schrag D, Downey R, Gelfand S, Begg C. The influence of hospital volume on survival after resection of lung cancer. N Engl J Med 2001; 345: 181 - 188

${ }^{3}$ Berdel WE, Eberhardt W, Passlick B, Rübe C, Thomas M (Hrsg). Contributions of the Conference „Interdisciplinary Treatment of Lung Cancer“. Lung Cancer 2001; 33 (Suppl 1): S1 - S164
} 